\title{
10
}

\section{Two approaches linking a test generation tool with verification techniques}

\author{
Marylène Clatin ${ }^{1}$, Roland Groz ${ }^{1}$, Marc Phalippou ${ }^{1}$, Richard Thummel ${ }^{2}$ \\ FRANCE TÉLÉCOM - CNET
}

\begin{abstract}
This paper presents two methods implemented in a test generation tool to compute significant feasible test paths including parameter values for input-output events. The first method is a kind of symbolic execution. The second method consists in linking the test generation tool with a tool permitting sophisticated types of reachability analysis. Preliminary results on non-trivial protocols are commented.
\end{abstract}

Keywords : conformance testing, protocol verification, Formal Description Techniques, test generation.

\section{Introduction}

Conformance testing has long been deemed a major issue of strategic importance for the acceptance and use of OSI and other products based on standards for open systems. ISO has established a methodology in its IS-9646 standard. The central point in a conformance testing process lies in the availability of a test suite which must be closely related to the protocol (or system) specification. Designing such test suites is hard work. Fortunately, the automatic generation of test suites is now becoming a reality, although existing tools are still limited.

Automatic test suite generation for protocols is based on a formal description, in languages such as Estelle, Lotos or SDL. The first goal of Formal Description Techniques (FDT) is to provide a precise and unambiguous description of a protocol. Based on such a description, verification techniques can be applied to check the consistency of a protocol specification with the corresponding service, or simply any protocol property. As a matter of fact, much effort has been devoted to protocol verification techniques, and many tools have been developed which take as input a specification in one of the three standard FDT. Interest in test generation from FDT is more recent, and has followed a different path so far.

Although the aims are different, there is a real interest in integrating verification techniques into test generation. This paper explores two approaches for solving a key problem in test generation. The problem, detailed below, is to find a feasible test path from one protocol state to another. The techniques considered are a kind of symbolic execution, and reachability analysis. Both approaches have been implemented in our test generation tool, called TVEDA, and we have been applying them to significant protocols.

Section 2 introduces the problem and the rationale for considering verification techniques; it also discusses some issues about our test generation environment. Section 3 describes the symbolic execution method used in our tool. Section 4 describes the other method, linking our tool with a powerful tool for reachability analysis. Section 5 discusses the pros and cons of each

1. CNET LAA/EIA/EVP, BP-40, F-22301 LANNION Cedex, FRANCE

Tel: 33960511 11, Fax: 33960539 45, E-mail: (clatin,groz,phalippo)@lannion.cnet.fr

2. Current address: Direction de l'aviation civile Sud, Aéroport de Toulouse, BP 100, F-31703 Blagnac Cedex

$\mathrm{R}$. Thummel contributed to the work reported here during his stay at Lannion for a collaboration between CNET and DGAC. 
method, based on our experiments with a few protocols, and gives some hints on key issues in the use of verification techniques for test generation.

\section{Basic issues}

\subsection{Time is ripe for linking test and verification}

Research in test generation techniques focused for a long time on specific issues which did not encompass the whole problem of generating a workable test suite from a formal specification. For instance, many papers in the 80s addressed the test architecture problems, others considered generation of check sequences for FSM etc. However, the development of tools generating test suites (esp. from FDT to TTCN) has led to a broader view of the problems of test generation. We have presented in [Groz 95] a list of problems, all of which are now addressed one way or another in the literature. There is therefore a better understanding of what is needed for generating tests. And some problems can be identified as sub-problems already addressed by verification.

At the same time, protocol verification tools are coming of age. Verification techniques are now implemented in commercial tools, and research prototypes are often strong enough to tackle real-size applications with sophisticated techniques.

Some work has already been done to connect test generation to existing verification tools. For instance, [Chun 90] uses a constraint solver to compute feasible paths traversing an EFSM (Extended Finite State Machine) specification; [Cavalli 92] uses a tool computing the reachability graph for a Lotos specification, in order to apply a modified version of the UIO method [Sabnani 88] on this graph. Both approaches suffer from complexity problems which limit their current applicability to small size specifications. Approaches proposed in commercial tools such as Topic [Montiel 94] or STED [Ek 93] offer also some connection between test generation (from already specified test purposes in this case) with simulation tools (not really verification in this case, but this is a step in the right direction). Although this paper takes a different approach, based on our experience with large scale applications, there is room for many fruitful interactions between test generation and verification tools.

\subsection{A key building block for test generation}

A test generation tool starting from an FDT-based specification and producing an abstract test suite (ATS) in, say, TTCN, must perform several functions which correspond logically to components of the tool. For instance, we presented in [Groz 95] the following global break up: getting an abstract test-oriented view from the specification, selecting test purposes, computing test case kernels, producing and formatting a complete test suite.

Test selection is specific to test generation, and pertains to research in testing. We do not expect verification techniques to be immediately applicable in that area. The same applies to the production of the final test suite (which takes into account such issues as test suite structure, TTCN format etc.).

Arguably, support could be found in verification techniques for the first step, that is to get an abstraction from the specification. In particular, it is necessary to abstract from events which cannot be observed or controlled from the tester, because of limits in the tester and in the test architecture. We did not (yet) consider verification techniques for this step, because it is not a time-intensive one in the case of our method, which abstracts a specification into an EFSM. 
Using efficient techniques might be more crucial in the case of methods translating specifications into less powerful abstract models, such as FSM or LTS [Cavalli 92][Chin 95], because in this case, the resulting abstraction is much larger, and efficiency is required to deal with it.

We have considered verification techniques for that step where efficiency in dealing with the semantics of the application is important, that is to say: computing test case kernels. Given some sort of test purpose, we would like to find which sequences of events are permitted by the specification and fulfil the requirements expressed by that test purpose. This is very similar to a verification problem because we are trying to verify the existence of some property in a specification.

As can be seen, this problem can be well defined (we will describe it in more detail below in section 2.5), and once defined, it is insulated from the rest of test generation procedures. The solver for this problem is a key building block for test generation. Since its interface with the rest of the test generation process can be well defined, solving it can be subcontracted to a verification tool.

Above all, it is important to note that this building block is generic enough to be used in several contexts. It can be used by different test selection strategies; for instance, we use it for computing transition subtours, or test preambles, or test postambles. It could be put to other uses.

\subsection{Combinatorial blow-up}

The main argument for resorting to verification tools is that the problem considered (identifying sequences of events satisfying a given property) raises combinatorial search with potentially an exponential blow-up. Research in verification techniques has investigated methods to limit that blow-up, and perform efficient searches.

In fact, an EFSM is a heavily factorized view of a protocol. The tests themselves must take into account the precise values of the variables which make up the Extended (E) part of the E-FSM. This means that the execution paths that form the kernel of tests are to be found in the FSM which would result from the full expansion of the EFSM. This FSM is in fact the reachability graph of the EFSM. The problem is that even for very simple protocols, this graph will be gigantic as soon as there are, for instance, several integer variables (e.g. frame numbering at link layer, even with frame numbers limited to a 256 modulo).

Our experience (and common experience) with various protocols and telecommunication services testifies that this problem will appear in most real applications, AND that it has a direct impact on various aspects of test generation. In some cases, finding which test paths are actually feasible can eliminate most proposed paths [Rouger 89]. Section 5 provides data on the complexity of the different protocols which we considered.

\subsection{Tool background}

Our first tool, called TVEDA-V2 (a previous version 1 - V1, was functionally similar, only the programming environment changed drastically), was based on a single strategy, which we called "test skeleton strategy" (based on the single step method of IS9646). This strategy produced TTCN tables corresponding to test cases, but did not produce the tables for preambles or postambles; also, some constraints (in the sense of TTCN) were not produced adequately in many cases because we considered each transition in isolation from its semantic context, i.e. disregarding the transitions that might have preceded it in a feasible execution path; as a consequence, we could not take into account the values of variables that were dependent on the path 
leading to this transition. Although this approach may seem limited, it can still generate the bulk of a test suite, as was presented in [Phalippou 90].

Our new tool, called TVEDA-V3 was partially presented in [Phalippou 94]. It incorporates several new features, apart from the fact that the design of the software is completely new from the previous version.

1. A modular architecture, that makes it possible to choose between: specification language (Estelle or SDL), test description language (Menuet [Langlois 89] or TTCN), test selection strategy (single transition, extended transition tour...)

2. A semantic module, which can be called from the strategy modules to compute feasible paths.

3. Sundry functional extensions, such as hypertext links between tests and specification (currently only for Estelle), test coverage analysis...

This paper deals with point 2 above. The idea is that this key point in computing test kernels can be implemented in different ways. We have investigated two approaches to implement this module: symbolic execution, and reachability analysis. Both approaches are actually implemented in TVEDA-V3, and the user of the tool can choose whichever of the two methods seems more appropriate for the protocol considered.

The modular approach to test generation makes it possible to plug into our tool this "semantic module", because the problem it addresses is orthogonal to the tasks addressed by other modules (such as output format, test strategy etc.).

\subsection{Precise definition of the problem addressed}

Before presenting the two approaches, let us state precisely the problem which they are both aimed at solving. In fact, the two solutions are very different in nature, applicability and efficiency, but they both compute the same result.

\section{Inputs:}

1. an EFSM, consisting of a base FSM with extended transitions; those extended transitions identify major from-state, to-state, input-event, provided condition on input parameters + internal variables, output-events, and assignments of new values to internal variables

2. a source domain, consisting of a major (FSM) state, and a condition (boolean expression) on the values of variables

3. a target domain, consisting of a major state, possibly an input event, and a condition on the values of variables (possibly linking them with input parameters)

\section{Goal:}

1. Compute at least one path from the source domain to the target domain, chaining transitions in accordance with the conditions specified by the EFSM.

2. Compute the non-determinism inherent in the specification, or due to limited controllability of the IUT (e.g. when the same input can trigger one of several transitions based on the availability of internal resources) or due to limited observability from the tester (when events on non observable channels can interfere). We called these last two types of non-determinism context non-determinism and event non-determinism respectively, in a previous paper [Phalippou 90]. This is very important to derive correctly the branches leading to inconclusive verdicts. In fact, in our modular tool, context and event non-determinism are already included in the EFSM as inherent non-determinism by the module which translates the specification (+ test architecture) into an EFSM. 


\section{Outputs:}

One solution tree (one = first-found, and shortest-path in fact for both solutions) consisting of

1. a sequence of chainable transitions of the EFSM; let us call this sequence a "path"

2. for each transition containing an input-event, a value for each parameter (such that it is consistent with the chaining)

3. for each transition, observable output events and their associated parameter values

4. for each transition, the list of interfering transitions (those that could fire in the same context as provided by the source domain of the transition AND the parameter values provided in 2 above)

Note: the problem stated above can be defined as the "single target problem". In fact, this basic problem is handled by our tool, but we also address the "multiple target problem": this is the case when the inputs include several targets, and we are trying to compute simultaneously one solution tree for each target. For instance, this happens for one of the most typical occurrence of our problem: compute a preamble for each transition of a specification. In that case, the source domain is the initial state, and we have as many targets as transitions. By computing simultaneously one path for each target, we can factorize the computations done on common prefixes.

\section{Symbolic computation technique}

In this section we present a first technique used for computing execution paths on extended automata: symbolic computation. We illustrate our explanations with an example based on an Estelle description of INRES protocol [Hogrefe 92]. However, the technique uses the «extended automaton» aspects of the language, and is also valid for SDL: in our tool TVEDA V3, it is implemented by using a preliminary translation of Estelle and SDL subsets into a common model based on EFSM. Figure 1 below describes the part of INRES Estelle specification which will be used (syntax is not strictly respected).

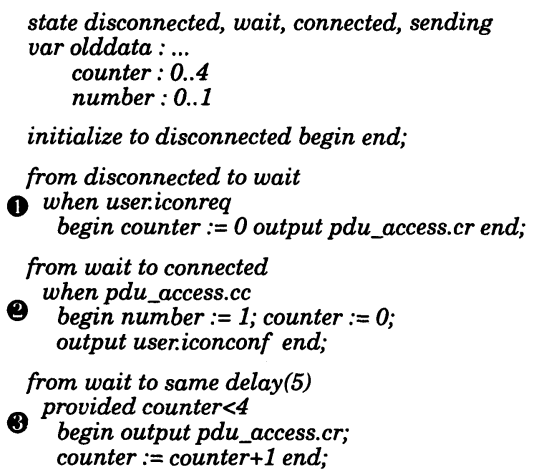

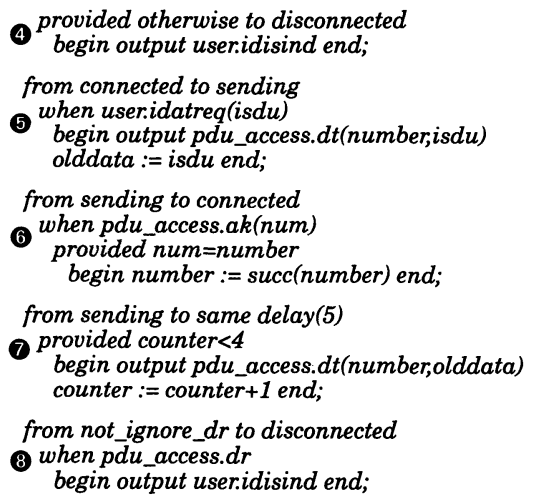

Figure $1:$ INRES specification (partial) ability graph of the FDT specification. We have explained in section 2.3 why we need to com- 
pute execution paths on this big automaton. Symbolic computation is a technique which allows to compute such execution paths without computing the big automaton. This allows to avoid the key problem of managing the size of such an automaton.

\subsection{Domain calculus on EFSM transitions}

The basic idea of symbolic computation is to avoid the enumeration of data values (as is done in reachability analysis). On the contrary, computation is performed directly on value domains: a domain is a compact representation of a set of data values (potentially big).

Estelle data structures (variables and message parameters) are transformed into elementary data vectors which can be of two types: integer interval (including the integer type) or enumerated type (including booleans). For doing this, composed types, such as arrays or records, are first expanded into elementary fields. Each elementary data vector has a name (built from the name of the corresponding Estelle data structure field) and can store an elementary value domain. An elementary value domain models a set of possible values for the elementary vector. The value domains are represented either by the constant value any (modelling all possible values of the given type), either by empty (no value) either by a list (of values for enumerated types, of intervals for integer interval types). The product of elementary vectors is called a vector, and we call value domain the product of elementary value domains. We use a vector to represent a set of states of the reachability graph, or a set of events (inputs or outputs). We show on figure 2 the vectors which represent respectively the set of states of the reachability graph of INRES after the initialize transition, and the set of when inputs of transition 6 of figure 1 . Note that the more any values appear in value domains, the more compact the representation is.

\begin{tabular}{|c|c|c|c|c|c|}
\hline \multirow{4}{*}{$\begin{array}{l}\text { states after } \\
\text { initialize }\end{array}$} & & el_vector1 & el_vector2 & el_vector3 & el_vector4 \\
\hline & \multirow{3}{*}{$\begin{array}{c}\text { name } \\
\text { type }\end{array}$} & state & olddata & counter & number \\
\hline & & enum. & enum. & interv. & interv. \\
\hline & & (disconnected) & any & any & any \\
\hline
\end{tabular}

\begin{tabular}{|c|c|c|c|c|}
\hline \multirow{3}{*}{$\begin{array}{c}\text { when inputs of } \\
\text { transition } 5\end{array}$} & \multirow{4}{*}{$\begin{array}{c}\text { name } \\
\text { type } \\
\text { value }\end{array}$} & el_vector1 & el_vector2 & el_vector3 \\
\hline & & pco & $p d u$ & $i s d u$ \\
\hline & & enum. & enum. & enum. \\
\hline & & (user) & (idatreq) & any \\
\hline
\end{tabular}

Figure 2 : Vectors and values

As mentioned above, symbolic computation means that we compute directly on domains. In order to compute transition paths on the reachability graph of the EFSM specification, we must be able to compute the effect of Estelle or SDL transitions on domains. This means:

1. compute the image domain which is reached after executing a transition if started from a given initial state domain and input domain.

2. compute the reverse image of a given final state domain (i.e. which initial state domain and input domain the transition must start from in order to ensure that the final state domain is reached). 
In order to do this, for each extended transition of the EFSM structure, we compute the following elements:

1. the guard of the EFSM transition (from state, when event and provided clause) is transformed into a couple of vectors (state vector and when vector) which represent the complete enabling domains of this extended transition.

2. the body of the EFSM transition (to state, assignment statements and output events) is transformed into a couple of procedures: the first one computes the image domain (final state domain, output domain) of the intersection of any initial domain (start state domain, input domain) with the (state vector domain, when vector domain) of the Estelle transition. The second one computes the reverse image domain (i.e. a start state domain and an input domain, possibly empty) of any final state domain.

These concepts are illustrated on figure 3 . We give two examples of reverse image domains. The first one is a normal case. The second one illustrates the case in which the final domain cannot be reached by the transition: in this case, the initial state and event domains are empty. Of course, this transformation can be done only if the EFSM (Estelle or SDL) satisfies some strong restrictions on the constructs which appear:

1. in the provided clauses no coupled constraints on several variables or parameters should appear: a vector domain must be the cartesian product of the domains of its elementary vectors. For instance provided $x<y$ where $x$ and $y$ are variables, is forbidden.

2 . in the transition body only constructs for which the image and the reverse image of domains can be computed are allowed. This includes a very limited number of expressions in assignment statements. For instance $x:=y$ is allowed (direct image: the domain value of $x$ after the assignment is the domain value of $y$ before. Reverse image: the domain value of $y$ before is the domain value of $x$ after, and the domain value of $x$ before is any). On the contrary, $x:=y+z$ is not allowed (impossible to compute the reverse domain as a product of elementary domains for $y$ and $z$ ).

In practice, many real protocol specifications satisfy such constraints, as explained in section 5 .

\subsection{Path computation}

We explain in this section how to use the domain calculus in order to compute the transition paths. As required in section 2.5, our goal is to find an executable transition path from a given start domain $D_{S}$ to a final domain $D_{F}$. We must add the requirement that they are domains in the sense of the previous section, i.e. products of elementary domains. The path computation algorithm has three steps:

1. computation of the shortest sequence of chainable extended transitions such that $D_{s}$ is included in the start domain of the first extended transition and $D_{F}$ intersects the final domain of the last extended transition. For this, we consider successively extended transition sequences of growing length, computing a direct propagation of value domains, using the image function mentioned in the previous section, starting from $D_{S}$. If we reach a domain which intersects $D_{F}$, we compute the intersection $D^{\prime}{ }_{F}$.

2. reverse propagation of $D^{\prime}{ }_{F}$ using the reverse image function mentioned in the previous section (this indicates from which subset $D^{\prime}{ }_{s}$ of $D_{s}$ we must start and which subsets of the successive when domains of the extended transitions sequence we must use in order to be sure to reach the final domain $D^{\prime}{ }_{F}$ ). 
start state vector

\begin{tabular}{|c|c|c|c|}
\hline state & olddata & counter & number \\
\hline (wait) & any & any & any \\
\hline
\end{tabular}

when vector

\begin{tabular}{|c|c|}
\hline$p c o$ & $p d u$ \\
\hline$\left(p d u \_a c c e s s\right)$ & $(c c)$ \\
\hline
\end{tabular}

three examples of the transformation functions applications

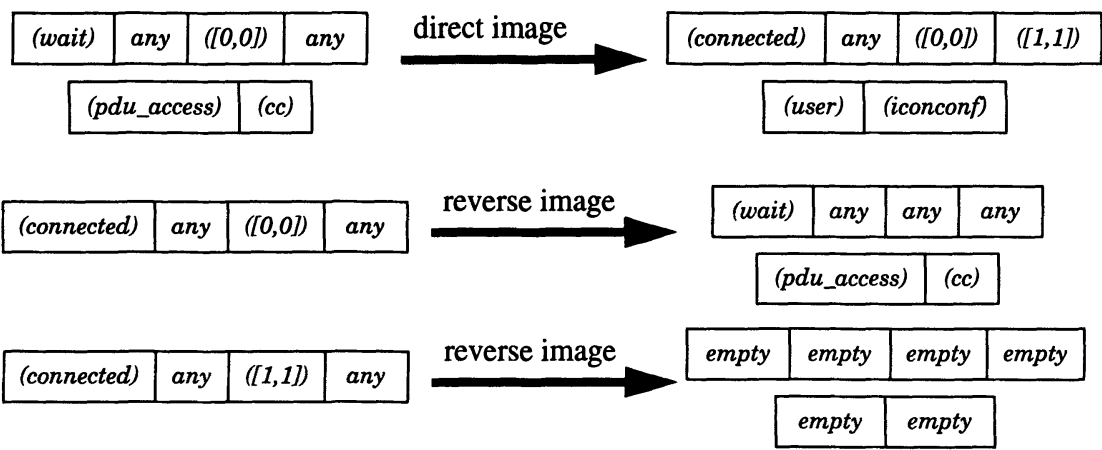

Figure 3 : Symbolic computation for INRES transition 2

3. arbitrary choice of values in the domains which result from step 2 , in order to obtain an instantiated transition path. Note that such an instantiated path is indeed a path on the accessibility graph which solves the first goal of the problem formulated in section 2.5.

Examples of path which are needed for test generation are preambles. A preamble for a given transition is a path which starts from the initial domain of the EFSM (i.e. the domain reached after the initialize transition, see figure 2) and reaches the when domain of the transition. Some preambles for INRES protocol, obtained by symbolic computation, are shown on figure 4 .

Path computation brings no additional restriction (other than the ones which are mentioned in the previous section) on the FDT constructs which are allowed. At this stage, the key point is performance, in order to deal with real protocols. Note that the vectors and the direct and reverse functions are computed from the compact EFSM model of the protocol: the size of such objects corresponds to the size of FDT descriptions, and avoids the size explosion which can be encountered when dealing with accessibility graphs.

The main performance problem comes from the fact that the number of chainable transition sequences grows exponentially with the length of the sequence, and the exponential factor depends on the number of transitions of the EFSM: the computation of long paths for big EFSM is a real challenge. Some optimization techniques have been implemented to improve efficiency: exact computations (e.g. detecting when a domain reached is included in an already reached domain), or heuristics (e.g. first reach a domain with the same major state as the target domain, then reach the exact target domain). 

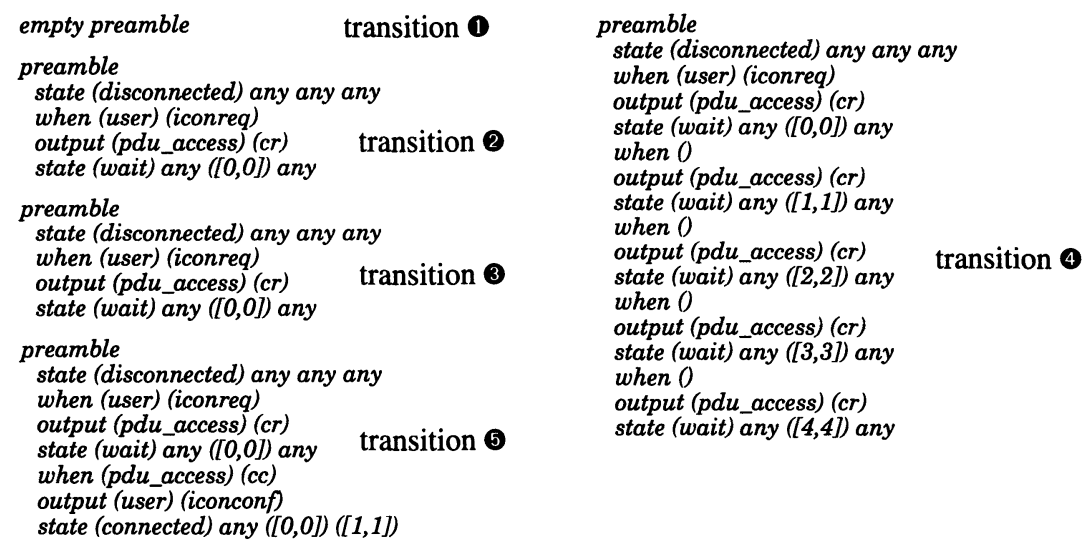

Figure 4 : Some preambles of INRES transitions (partial)

\subsection{Non-determinism computation}

Finally, the last step is the computation of the effect of non-determinism. According to the requirements of section 2.5, we must compute the effect (output behaviour and state reached) of every transition which can be non-deterministically fired with any transition of the path we have computed. For doing this, we compute the successive domains which are reached starting from the initial domain of the EFSM and using the input values which have been chosen during step 3 of the path computation procedure. For each of these successive domains, we compute the firable transitions (either spontaneous, or with the same input as in the path), and their output and final state domains. Adding non-determinism, the preamble sequences of figure 4 are transformed into the test trees of figure 5. Note that only the preamble of transition 6 contains a non-deterministic event, leading to an inconclusive verdict.
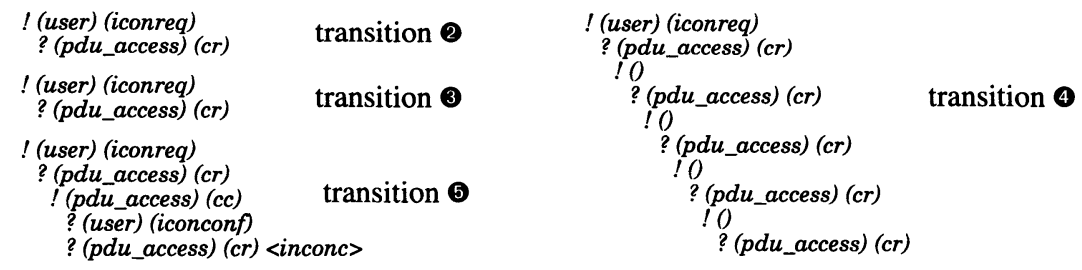

Figure 5 : Test trees obtained from the preambles

\section{Reachability technique}

Another way of computing transition chains consists in using a simulator. In our case, we have coupled TVEDA with Véda. Véda is a tool which has been designed for the specification and 
verification of systems described with a subset of the ISO Estelle language. Its strength relies mainly on its powerful simulation and verification abilities. Indeed it supports interactive and random simulation techniques as well as formal verification based on reachability state graph analysis. Véda was developed at CNET and industrialized by Vérilog [Algayres 93].

TVEDA is coupled with Véda in the following way. First, TVEDA builds an extended finite state machine (EFSM) from the Estelle or SDL specification given in input. Then Véda is used in three steps:

1. First, this EFSM is transformed so as to reduce its size, while preserving the existence of paths. The transformed EFSM is decompiled into Estelle (the input language of Véda).

2. Then we use three heuristics to address the multiple target problem as stated in section 2.5 . Each heuristic allows to compute some of the paths:

a. an exhaustive simulation is made. Duration of the exhaustive simulation is limited, so as not to spend too much time waiting for the results. During this first step, a major part of the paths is found.

b. a second step consists, for each transition not reached during the first step, in making an exhaustive simulation using a distance computation between states. When the distance is too large, the simulation is given up. Some other paths may be found during this second step, but possibly not all.

c. Finally the last step consists in loading a path which brings the specification in a state close to the initial state of the transition, and then in making an exhaustive simulation. The simulation runs until the transition is reached.

3. Finally, these paths have to be completed to integrate non-determinism and constraint values.

We detail now these three steps.

\subsection{EFSM transformations}

There are two reasons which motivate the EFSM transformations. First, we want to have a specification which can be simulated using Véda, without adding any environment to generate input events. Secondly, we want to eliminate all things which are not really needed for path computation in order to improve the efficiency of the reachability analysis.

The EFSM transformations are the following ones:

1. hiding of the environment (all transitions become spontaneous, output events are suppressed),

2. computation and treatment of the specification «useful part» and suppression of the useless part. The useful part is defined as the specification elements which influence transition firing (variables and parameters which appear in provided clauses, and therefore have an influence on transition firing, and transitive closure on parameters and variables for assignments),

3. grouping transitions into equivalence classes, after reductions 1 and 2 . A class contains all transitions which become equal when the useless part is suppressed.

These transformations are illustrated on figure 6. From the specification described on figure 1, we obtain this latter after transformations.

The EFSM obtained after transformation is different from the EFSM we had initially, but they both have the same behaviour in terms of possible state sequences (a state is defined here by the global state of the specification and the value of variables computed as «useful»). Input 


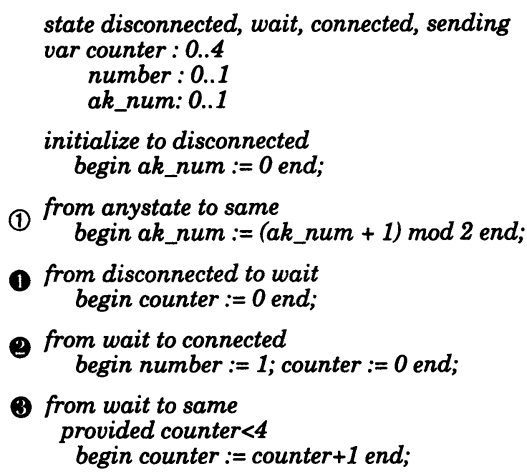

(4) provided otherwise to disconnected begin end;

5 from connected to sending begin end;

6 from sending to connected provided $a k \_$num $=$number begin number := succ(number) end

$(7$ from sending to same provided counter $<4$ begin counter := counter +1 end;

8 from not_ignore_dr to disconnected begin end;

Figure $6:$ INRES specification (partial) after transformations

events have been suppressed, but their useful parameters are kept and modelled as variables which evolves thanks to special transitions added to the EFSM (on the above example, num parameter of $a k$ interaction is a useful parameter modelled as a $a k \_n u m$ variable. The added transition is (1)). Therefore, there is no longer interaction with the environment, since the new specification is completely autonomous.

Then, in order to compute paths, we simulate the specification obtained by decompiling the transformed EFSM into Estelle, and the computed paths on this specification are still valid paths for the initial one.

\subsection{Path computation heuristics}

As indicated in the introduction to section 4, we use three consecutive heuristics to compute a path for each target. This is required because none of the three heuristics is powerful enough to find all paths. We try, successively:

1. Bounded searching: this first step consists in making an exhaustive simulation of the specification, restricted by the number of states of the reachability graph. Since the exhaustive simulation is based on building a reachability state graph, when the state number is reached the simulation ends. For simple specifications (such as Inres protocol) all paths are found during this phase, and for more complex ones (such as LAPD protocol) only $80 \%$ are found, because the reachability graph is too big to be entirely computed. Due to breadth exploration of the reachability graph, the path found for each transition constitutes the shortest path from the starting state to the target.

2. Searching based on a distance computation: for each transition not reached during the first step, a second heuristic is used which consists in performing another exhaustive simulation taking into account a distance between states. Such empiric distance models the «difference of values» between two states. The distance must decrease during the path exploration (more or less strictly). If the distance increases, the current path exploration is given up, and searching goes on another branch of the graph. At the end of this second step, some other paths may have been computed in addition to those found during the first step.

3. Searching using a path prefix: finally, for each remaining transition not reached during the last step, a third heuristic consists in loading a prefix which brings the specification in a state close to the transition initial state (with good variable values satisfying the transition guard 
predicates). After this prefix loading, an exhaustive simulation happens with no restriction on its duration.

At the end of this phase, we have computed paths in the shape of transition names lists. These paths correspond to transitions of the transformed EFSM. Since the transformed EFSM and the initial one have the same behaviour in terms of possible states sequences, a simple translation on the transition names lists is performed to get paths for the initial specification. Basically, it consists in choosing one original transition for each equivalence class. So far, we have reached the first goal («output» 1 in section 2.5$)$. We must now compute the other outputs $(2,3$ and 4$)$.

\subsection{Integrating non-determinism and constraint values}

Here again, Véda is helpful. Indeed, each path obtained during the last phase is played on the initial specification which is slightly transformed so as to give us the lacking informations. Some of these informations are directly given by Véda, whilst some others have to be computed. This is the reason why we add some extensions to the initial specification.

When playing a path (called scenario in Véda's terminology) on a specification, Véda lists at each scenario step the set of firable transitions. Non-determinism is then obtained at each step from the list of firable transitions and the fired transition name. When playing a scenario, Véda also lists message emissions and the value of each message parameter. Then, informations for constraint values concerning message emissions are directly given par Véda. Concerning message receptions we cannot get directly the informations, because environment has not been modelled. An extension to the specification allows to solve this problem, as described hereafter.

The specification used in this part is obtained from the initial specification, modelled as an EFSM, which is then copied before being transformed. Some reductions are performed so as to simulate the environment; the main reduction makes all transitions spontaneous. Message receptions are found by searching in the initial EFSM the transition thanks to its name (transition names are kept during EFSM copy). So, the last problem concerns reception message parameters. This is solved in the following way. First each parameter is declared as a variable. Then for each parameter belonging to the useful part the same treatment as performed in section 4.1 is done. And for each parameter belonging to the useless part, we give it a value which satisfies the corresponding transition guard by means of another transition (fired just before the one we talk about). All these transformations are illustrated on figure 7.

The reduced and extended EFSM is decompiled in Estelle and simulated with Véda by playing each scenario found during the last phase. A trace file is then produced by Véda, containing all informations relevant to test generation including non-determinism and constraint values.

An example of test generation using this technique is described on figure 8. This figure represents the preamble produced for transition 6 of the initial specification (cf. figure 1) in a TTCN-like tree notation.

\section{Assessment and perspectives}

After the presentation of the techniques we used, we conclude now this paper with an assessment of the results that have been reached so far, and we conclude with some words about the future development perspectives.

As we mentioned before, both techniques (symbolic computation and reachability analysis) have been implemented in our tool TVEDA V3, as a means for computing extended transition 


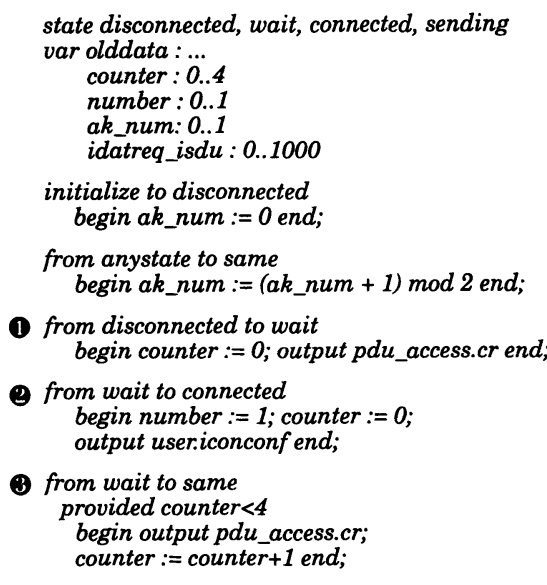

4 provided otherwise to disconnected begin output useridisind end;

from connected to connected begin forone idatreq_isdu_var:0..1000 suchthat true do begin idatreq_isdu $:=$ idatreq_isdu_var end end

5 from connected to sending begin output pdu_access.dt (number,idatreq_isdu) olddata := idatreq_isdu end;

6 from sending to connected provided $a k_{\_}$num $=$number begin number $:=$ succ(number) end

8 from sending to same provided counter $<4$ begin output pdu_access.dt (number,olddata) counter : = counter +1 end;

8 from not_ignore_dr to disconnected begin output user.idisind end;

Figure 7 : INRES specification (partial) reduced and extended

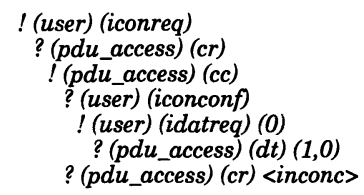

Figure 8 : Test case obtained for transition 6

tours [Phalippou 94] on Estelle or SDL specifications. However, the optimization techniques which are listed previously are not completely operational: for this reason the figures we give here will probably be enhanced later on.

\subsection{Symbolic computation}

We mentioned in section 3 that this technique has two kinds of limitations: it puts some strong restrictions on which constructs are accepted (see section 3.1) and the path computation requires an exponential computation with respect to the length of the path to be computed.

Surprisingly (when we think how strong are the limits on constructs) the first point is not really a problem for many protocols: although many SDL or Estelle specifications considered had some forbidden constructs, in most cases, it has been possible to rewrite the specification to avoid them. This means that the use of such constructs is not linked to the mechanisms of the protocol themselves, but rather to specification styles of the FDT programmer. Among successfully tried protocols, let us mention INRES (Estelle and SDL versions), ISDN D signalling protocol, P1 (a MHS X400 series protocol), FRP and SSCOP (ATM protocols), some supplementary serviced provided on ISDN networks, service descriptions used in Intelligent Network. 
As a counter-example, ISDN D link-layer protocol (LAPD) cannot be completely processed, since it incorporates a sliding window mechanism, which cannot be modelled without some provided clauses incorporating coupled variable values: in that case the state vector cannot be represented as a cartesian product of elementary vectors.

About performance during path computation, we obtained the following results:

1. on simple protocols, such as INRES, there is no problem: all paths are computed within some minutes (on a SPARC LX workstation). This result is valid for all small examples we tried, e.g. EFSM up to 50-60 transitions. Let us note however that the exponential time computation is verified: in a very particular case (an EFSM with only 31 transitions, but paths of length up to 15 , which is quite big) it took 25 hours on the same machine (in the case of INRES, the maximum length of the paths was 7).

2. on big protocols with (relatively) simple data structure, such as ISDN D protocol, it is possible to compute only short paths (depth 3 in the case of D signalling protocol, which has about 2000 transitions). Of course this is only a very limited subset of the paths to be computed. However, after a (human) analysis of the protocol structure, it is sometimes possible to split the specification into several parts which are independent as far as path calculus is concerned: with such a technique, we succeeded in the computation of $2 / 3$ of the 765 test cases of the LAPD protocol.

3. on big protocols with complex data structures, such as P1, we could not compute the internal representation of vectors, due to memory limitations. As an indication, the state vector has more than 1500 elementary vectors, the when vectors are up to 250 elementary vectors, and there are 115 transitions to be modelled!

In conclusion, the symbolic computation technique, as it is now in TVEDA, is adapted for the treatment of small specifications, but fails when the EFSM become large. The technique by itself seemed promising, since it can be potentially applied to EFSM with big (even infinite) data domains. However, on the studied examples, this did not appear to be a key point (no real protocol has infinite domains!). On the contrary, the weakness of our approach may come from the fact that we developed ourselves an ad-hoc implementation of the method (not at all optimized from a performance point of view), mainly because we could not find such an industrial tool available.

\subsection{Reachability analysis}

On the contrary, reachability analysis presents two advantages:

1. the technique puts almost no restriction on the Estelle or SDL constructs which are accepted (which means that all our example specifications can be potentially handled by this technique),

2. it is implemented by interfacing our tool TVEDA with a powerful commercial tool for reachability analysis, Véda.

We have not yet finished to implement all optimization features which have been described in section 4.2. However, the results we have so far are promising:

1. on simple protocols such as INRES, all paths are computed within some minutes (in such simple cases, most of the computation time is spent in the compilation step with Véda. Path calculus through Véda execution is almost instantaneous).

2. on LAPD case study, about $80 \%$ of the paths needed for the test cases are computed, using only the bounded searching heuristic for path computation (the other heuristics have not been implemented so far). We have good hopes to be able to compute almost all needed 
paths once the other heuristics are implemented, as was proved on LAPD in a semi-automatic implementation of heuristics 2 and 3.

From these results we conclude that reachability analysis will probably succeed in solving our path computation problems, at least for some kinds of protocols. However, we must still investigate with different specifications (e.g. P1, which incorporates very big data structures). Moreover, in the case of LAPD, and in spite of all the optimization features that have been incorporated in our technique, we are very close to the limitations of Véda tool (size of the Estelle source that can be accepted at compilation step, for instance). Note that the computation of the preambles took more than 8 hours on our workstation (mainly spent during the compilation step of the simulator).

\subsection{About the use of verification techniques}

Verification techniques seem to be promising candidates for solving key points in the test generation process. However, their successful use for this purpose will depend on some crucial aspects that have been highlighted by our experiments:

1. the verification technique must have reached a good level of maturity, and should be implemented in a tool of industrial-level efficiency. This was the case for our experiment with reachability analysis, but not with symbolic computation, and this may explain the (relative) failure of the latter.

2. industrial tools for verification have been developed for verifying protocols, not for providing help to test generation tools. Therefore their interfaces must be adapted. For the moment, this leads to tricky interface programming (see the link between TVEDA and Véda described in this paper). In the future, we can imagine that verification tools will evolve towards more open and generic interfaces.

3. even if condition 1 is fulfilled, careful (and sophisticated) optimization must be done in order to be sure that the computation problem submitted to the verification tool is simplified as much as possible (e.g. the computation of the «useful part» of the specification, or the modelling of environment modules in our implementation). Without such optimizations, handling big specification may still be beyond the strength of the tools.

4. a combined use of different techniques (each one with its strengths and weaknesses) may be useful to cope with very different specification styles, and application domains. Several techniques could be potential candidates for further studies in this direction. Let us mention for instance constraint solving techniques, abstract interpretation [Cousot 77], or a mixture of abstract interpretation with reachability analysis.

\section{References}

[Algayres 93]

B. Algayres, L. Doldi, H. Garavel, Y. Lejeune, C. Rodriguez, VÉSAR: a pragmatic approach to formal specification and verification, Computer Networks and ISDN Systems, special issue on tools for FDTs, vol. 25, n. 7, February 1993.

[Cavalli 92]

A. Cavalli, Sung Un Kim, Automated protocol conformance test generation based on formal methods for LOTOS specifications, proceedings of the 5th International Workshop on Protocol Test Systems, Montréal, September 1992.

[Chin 95]

B-M. Chin, A. Cavalli, Test generation methods for SDL, tutorial proceedings of SDL forum 95, Oslo, September 1995. 
[Chun 90]

W. Chun, P. Amer, Test Case Generation for Protocols Specified in Estelle, actes de FORTE'90, Madrid, novembre 1990, Elsevier, pp 191-206.

[Cousot 77]

P. Cousot, R. Cousot, Abstract interpretation: a unified lattice model for static analysis of programs by construction or approximation of fixpoints, 4th POPL, January 1977.

[Ek 93]

A. Ek, J. Ellsberger, A. Wiles, Experiences with computer aided test suite generation, proceedings of IWPTS 93, Pau, September 1993.

[Groz 95]

R. Groz, M. Phalippou, La génération automatique de tests est-elle possible ?, proceedings of CFIP'95, Rennes, May 1995.

[Langlois 89]

C. Langlois, E. Paul, De la spécification formelle à la validation de systèmes, actes du deuxième colloque annuel du club FIABEX, INT-Évry, 22-23 novembre 1989.

[Montiel 94]

J. Montiel, R. Roth, A.J.M. Donaldson (Eds), Methods for QoS Verification and Protocol Conformance Testing in IBC - Results and Further Recommendations, Race Projet R2088 TOPIC, Deliverable 15, Doc R2088/DAT/TMS/DS/P/015/b1, November 1994.

[Phalippou 90]

M. Phalippou, R. Groz, Evaluation of an empirical approach for computer-aided test cases generation, proceedings of the 3rd IWPTS, Washington, October 1990.

[Phalippou 94]

M. Phalippou, Test sequence generation using Estelle or SDL structure information, proceedings of FORTE'94, Bern, October 1994.

[Rouger 89]

A. Rouger, P. Combes, Exhaustive validation and test generation in ELVIS, proceedings of the $4^{\text {th }}$ SDL forum, North Holland, 1989.

[Sabnani 88]

K. Sabnani, A. Dahbura, A Protocol Testing Procedure, Computer Networks and ISDN Systems, vol. 15, n. $4,1988$. 